\title{
Male breast cancer: Specific biological characteristics and survival in a Portuguese cohort
}

\author{
SAUDADE ANDRÉ ${ }^{1}$, TERESA PEREIRA $^{1}$, FERNANDA SILVA ${ }^{2}$, PATRÍCIA MACHADO $^{3}$, \\ FÁTIMA VAZ ${ }^{3}$, MARIANA APARÍCIO ${ }^{4}$, GIOVANI L. SILVA ${ }^{4,5}$ and ANTÓNIO E. PINTO ${ }^{1}$ \\ ${ }^{1}$ Department of Pathology, Portuguese Institute of Oncology of Lisbon, 1099-023 Lisbon; \\ ${ }^{2}$ NOVA Medical School, NOVA University, 1169-056 Lisbon; ${ }^{3}$ Breast Cancer Risk Evaluation Clinic, \\ Portuguese Institute of Oncology of Lisbon, 1099-023 Lisbon; ${ }^{4}$ Department of Mathematics of Higher Technical Institute, \\ University of Lisbon; ${ }^{5}$ Statistics and Applications Center of University of Lisbon, 1049-001 Lisbon, Portugal
}

Received September 19, 2018; Accepted March 29, 2019

DOI: $10.3892 / \mathrm{mco} .2019 .1841$

\begin{abstract}
Male breast cancer (BC) represents an individual subtype of $\mathrm{BC}$, with therapeutic procedures based on female BC therapy results. The present study evaluated the parameters currently used for the characterization and therapy of male BC, and their association with disease-free (DFS) and overall survival (OS), aiming to obtain a comprehensive basis to improve the personalized care of male BC. A total of 196 patients from March 1970 to March 2018 (mean follow-up, 84.9 months) were profiled, using clinicopathological review, molecular assessment [BRCA1/2, DNA repair associated (BRCA1/2) status, immunohistochemistry, fluorescence in situ hybridization and DNA flow cytometry] and Cox regression statistical analysis. The median age of patients was 66.5 years. At presentation, $39.2 \%$ of patients with invasive carcinomas were in anatomic stage (AS) I. Patients exhibited primarily invasive carcinomas of no special type, histological grade 2, estrogen receptor $\alpha$-(ER $\alpha)$ and progesterone receptor (PR)-positive, receptor tyrosine kinase erbB-2-negative, high Ki-67, Luminal B-like and aneuploid tumors. A total of 13 of the $44(29.5 \%)$ BRCA-evaluated patients exhibited BRCA2 mutations, significantly associated with family history (FH), bilaterality, high Ki-67 expression, absence of PR and Luminal B-like tumors. Bilaterality was associated with the occurrence of non-breast primary neoplasms (NBPN). The 5 and 10-year DFS rates, excluding patients with distant metastasis, NBPN and in situ carcinomas $(\mathrm{n}=145)$ were 65.9 and $58.2 \%$, respectively, and the 5 and 10-year OS rates were 77.5 and $59.2 \%$, respectively. In the univariate analysis, Luminal B-like subtype, BRCA2 mutations, high Ki-67 expression, and AS II
\end{abstract}

Correspondence to: Dr Saudade André, Department of Pathology, Portuguese Institute of Oncology of Lisbon, Professor Lima Basto Street, 1099-023 Lisbon, Portugal

E-mail: sandre@ipolisboa.min-saude.pt

Key words: male breast cancer, clinicopathological characteristics, molecular features, survival and III were significantly associated with shorter DFS and OS. In addition, age $>70$ years was associated with low OS. In the multivariate analysis, FH, AS II and III, and Luminal B-like subtypes were associated with poorer OS. In conclusion, the data from the present study emphasize the high incidence of BRCA2 mutation in male $\mathrm{BC}$, and its association with $\mathrm{FH}$, bilaterality, high Ki-67 expression, negative PR expression and Luminal B-like subtypes, and with shorter DFS and OS in univariate analysis.

\section{Introduction}

Breast cancer (BC) is a heterogeneous and complex disease, with a great variation in clinical outcomes. BC is the most frequently diagnosed cancer in females and second in causes of cancer mortality in both sexes, as metastatic $\mathrm{BC}$ remains an almost incurable disease. Incidence of male $\mathrm{BC}$ is rare $(\sim 1 \%$ of numbers of female $\mathrm{BC}$ and $1 \%$ of all malignancies in males in Western countries); however, in previous years, a slight increase in incidence has been observed in certain countries (1-5). BC in males has become most frequently diagnosed at an early anatomic stage (AS), and an improvement in overall survival (OS) has been observed (6-9). However, the lack of information regarding male $\mathrm{BC}$ and the unviability of screening have contributed to a persistently high percentage of diagnoses at advanced AS. In Portugal, the annual male BC gross incidence rates in 2010 and 2011 were 1.23 and 1.77 per 100,000 people, respectively, and the gross mortality rates were 0.34 and 0.51 , respectively (10).

Despite increasing interest, the biology and optimal management of male $\mathrm{BC}$ remain poorly understood, and contradictory data are often identified. Certain common epidemiological risk factors, which remain to be identified in either sex, may be relevant in the understanding and prevention of the disease (11). Genetic predisposition appears to be an important contributor to risk and may have clinical implications $(8,12,13)$. Family history $(\mathrm{FH})$ is also relevant, and a positive $\mathrm{FH}$ in a masculine family member is strong indication for genetics consultation (13). In addition, genetic mutations may be identified in patients without $\mathrm{FH}$ and should be routinely screened in male BC (13). In contrast to those 
identified in females, mutations in the BRCA2, DNA repair associated (BRCA2) gene are predominant in male BC, while BRCA1, DNA repair associated (BRCA1) gene mutations are infrequent $(8,12,14,15)$.

Obesity is one of the most common causes of hyperestrogenism in males, and adolescent overweight has been associated with an increased risk of male BC $(2,12,16)$. In addition, liver diseases, alcoholism, Klinefelter's syndrome, exogenous estrogen use (namely for the treatment of prostate cancer) and androgen deficiency due to testicular disease including hypogonadism and orchitis, appear to increase the risk of disease $(2,4,7)$. Occupational and environmental exposures to radiation, and heat and electromagnetic fields have also been implicated as potential risk factors $(3,8,12)$.

Male BC is diagnosed by mammography or ultrasonography and confirmed by core biopsy, which are always performed following a suspicious clinical examination. Therapeutic procedures are based on the recommendations for female $\mathrm{BC}$, but mastectomy rather than breast-conserving surgery is performed in the vast majority of cases. In addition, hormone therapy is less tolerated in males compared with in females, and side effects including weight gain, depression, deep venous thrombosis, decreases in libido and impotence, with high rates of discontinuation, were described $(4,5,17,18)$.

Molecular testing in BC, through the use of sophisticated techniques including deep sequencing analysis, has led to an improved understanding of this disease. Concomitantly, the identification of targeted therapies has reinforced the requirement for improved stratification in BC subtypes (13). The present study investigated a retrospective series of male $\mathrm{BC}$ cases, assessed the clinicopathological and molecular characteristics that are currently the basis for therapeutic strategies, and estimated their association and significance in disease-free survival (DFS) and OS of male BC.

\section{Patients and methods}

Patient selection and clinicopathological evaluation. The present retrospective study involved 196 male patients with BC diagnosed and treated according to therapeutic protocols from March 1970 to March 2018 (mean follow-up time, 84.9 months), at the Portuguese Institute of Oncology of Lisbon (Lisbon, Portugal). The institutional Ethical Committee of the Portuguese Institute of Oncology of Lisbon approved the study. Patient data, including age, obesity, $\mathrm{FH}$, bilaterality, presence of non-breast primary neoplasms (NBPN), presence of distant metastasis and therapeutic modalities were obtained by review of the clinical records. All slides were reviewed. AS classification included pathological tumor size (pT), pathological axillary nodal status $(\mathrm{pN})$ and distant metastasis (M), and was registered according to the TNM classification system recommended by the 8th edition of the American Joint Committee on Cancer (AJCC) staging system (19). The histological type was re-evaluated as per the World Health Organization 2012 classification (20). Histological grade of differentiation $(\mathrm{G})$ was assessed using the Elston-Ellis grading system criteria (21).

BRCA status. Nucleic acids were obtained from peripheral blood nucleated cells. DNA was extracted with the EZ1 Bio
Robot and the EZ1 DNA blood kit (Qiagen GmbH, Hilden, Germany) and RNA was extracted using TRIzol ${ }^{\circledR}$ (Thermo Fisher Scientific, Inc., Waltham, MA, USA). A total of 44 patients were pre-screened for the c.156_157insAlu $B R C A 2$ Portuguese founder mutation, analyzed for BRCA1/2 point mutations by next generation sequencing (NGS) with a CE-IVD MASTR BRCA molecular diagnostic assay (Multiplicom; Agilent Technologies, Inc., Santa Clara, CA, USA) in a MiSeq Instrument (Illumina, Inc., San Diego, CA, USA) and for large rearrangements with a Multiplex Ligation-dependent Probe Amplification (MLPA) assay using P002 BRCA1 and P045 BRCA2/CHEK2 kits (MRC-Holland, Amsterdam, The Netherlands). Variant Studio v.2.2 (Illumina, Inc, San Diego, CA, USA) and DNAnexus, Inc, Mountain View, CA, USA were used to analyze the NGS data, and Coffalyzer (MRC-Holland, Amsterdam, The Netherlands) software was used for the MLPA data. In addition to the information provided in the Variant Studio and DNAnexus programs, the Breast Cancer Information Core database and the Universal Mutation Database were used to clarify certain variants. Prior to NGS screening, patient samples were analyzed by conformation sensitive capillary electrophoresis or conformation sensitive gel electrophoresis, and samples with different patterns (fragment pattern comparison between the 44 patient samples analyzed in the same batch and also comparison with a negative control) were sequenced by Sanger sequencing using an ABI 3130 instrument as described previously $(22,23)$.

Estrogen receptor $\alpha(E R \alpha)$, progesterone receptor $(P R)$, receptor tyrosine kinase erbB-2 [(ERBB2), antigen $\mathrm{Ki}-67$ (Ki-67) immunohistochemistry (IHC) and ERBB2 in situ hybridization (ISH)]. IHC was performed using a peroxidase-indirect-polymer technique performed on a Ventana Benchmark $^{\mathrm{TM}}$ ULTRA instrument (Ventana Medical Systems, Inc.; Roche Diagnostics, Basel, Switzerland) on formalin-fixed paraffin embedded tumor tissues. All cases were re-analyzed under the same conditions for all samples within this study and all kits were used according to the protocol of the manufacturer. The levels of ER $\alpha$ clone SP1 (Ventana Medical Systems, Inc.; Roche Diagnostics; cat. no. 790-4324) and PR clone IE2 (Ventana Medical Systems, Inc.; Roche Diagnostics cat. no. 790-2223) were recorded as the percentage of positively-stained neoplastic cell nuclei, using a $\geq 1 \%$ cut-off value as the criterion for positivity. The staining intensity was not evaluated (24). The ERBB2 clone 4B5 (Ventana Medical Systems, Inc.; Roche Diagnostics cat. no. 790-2991) was used for ERBB2 evaluation. The quantification of ER $\alpha$, PR and ERBB2 oncoprotein overexpression $(0,1+, 2+$ and $3+$ ) was based on the American Society of Clinical Oncology (ASCO)/College of American Pathologists (CAP) guidelines (25). The Ki-67 index was recorded as the percentage of positively-stained cells, using the Ki-67 clone 30-9 (Ventana Medical Systems, Inc.; Roche Diagnostics; cat. no. 790-4286), and 'hot spots' were classified as those areas containing 100 malignant cells. The threshold for high proliferation was $20 \%$, based on the adaptation of the 2013 St. Gallen consensus guidelines (26). ERBB2 gene amplification was determined by FISH using a BenchMark ${ }^{\mathrm{TM}}$ Ventana $^{\circledR}$ system (Ventana Medical Systems, Inc.; Roche Diagnostics) or, in samples collected from 2009, by silver in situ hybridization 
(SISH) with evaluation of chromosome 17 (Dual-Color SISH red ISH/Benchmark ${ }^{\mathrm{TM}}$ ULTRA Ventana ${ }^{\circledR}$; Ventana Medical Systems, Inc.; Roche Diagnostics) in 77 cases, which included 38 IHC-negative (0/1+), 35 equivocal (2+) and 4 positive (3+) cases, according to the latest ASCO/CAP guidelines. The IHC pattern, complemented with ERBB2 ISH, allowed the identification of clinically-defined, treatment-oriented subtypes (CS), according to AJCC, 8th Edition (19), and were as follows: Luminal A-like (high hormone receptors and low proliferation level), Luminal B-like (low hormone receptors and high proliferation level), HER2-like (ERBB2-positive and negative or positive hormone receptor expression) and Triple negative (TN; ER-, PR- and ERBB2-negative).

DNA flow cytometry. DNA flow cytometric analysis was performed on representative paraffin-embedded tissue according to the method described by Hedley et al (27), with slight modifications $(50 \mu \mathrm{m}$ sections and propidium iodide DNA staining were used). DNA content of the neoplastic cells was determined in 79 invasive carcinomas with no neoadjuvant therapy. The cell cycle analysis of DNA histograms was assessed using the Multicycle software program (32-bit version; Phoenix Flow Systems, San Diego, CA, USA). The male BC cases were also classified as diploid vs. aneuploid according to their nuclear DNA ploidy status.

Statistical analysis. A survival study with an initial descriptive analysis and subsequent nonparametric, semiparametric and parametric statistical techniques was elaborated. The statistical analysis was performed using the software R Core Team 2018 (28). Pearson's $\chi^{2}$ and Fisher's exact tests of independence were used to evaluate the association between categorical variables. Fisher's exact test was used when the number of observations was small $(\mathrm{n}<20)$. Identical conclusions were obtained following the use of each test. Survival curves were estimated using Kaplan-Meier analysis, and the differences between curves were assessed by the log-rank test. $\mathrm{P}<0.05$ was considered to indicate a statistically significant difference. The DFS represented the remission time until a relapse event. The OS was defined as the interval between pathological diagnosis and the occurrence of mortality due to BC. Patients without disease recurrence during the study period and those who succumbed to other causes, or those who were lost to follow-up, were considered as censored observations. The OS was evaluated in the subgroup of patients with M1 disease, and the DFS and OS in the whole series, excluding patients with M1 disease, NBPN and in situ carcinomas. OS and DFS were also evaluated and compared in the following groups: Patients with vs. patients without NBPN, and patients diagnosed in the years 1970-1998 (group $A ; n=84$ ) vs. those diagnosed in the years 1996-2018 (group B; $n=132$ ) (prior and subsequent to the introduction of taxane chemotherapy). The variables describing the type of treatment were excluded due of the variability of protocols used. A Cox proportional hazards regression model was employed to assess the independent prognostic value of the variables. Initially, the model was fitted for each variable to evaluate their effects on OS time and remission time of disease, as a simple regression analysis. Following the determination of significant variables, a Cox regression model was performed with all variables simultaneously, as a multiple regression analysis. A backward stepwise procedure based on Akaike's Information Criterion (29) was used to select auxiliary variables. The statistical significance was obtained by the Wald test, and complementary inference was calculated as relative risk and $95 \%$ confidence intervals for each category. The quality of the fitted models was assessed using a residual analysis, and a test of proportionality of risk functions was conducted for Cox regression models associated with OS and DFS.

\section{Results}

Descriptive analysis and associations. The clinicopathological and therapeutic characteristics of the series are summarized in Table I. BRCA mutation, hormone receptors and $\mathrm{Ki}-67$ protein expression, ERBB2 overexpression and amplification, CS and DNA ploidy data are presented in Table II. Significant associations between variables are indicated in Table III.

The mean and median age of patients at diagnosis was 65.2 and 66.5 years (range, 31-89 years), respectively. The majority of the patients ( $\mathrm{n}=108 ; 55.1 \%)$ were between 40 and 69 years, and older patients ( $\geq 70$ years) comprised $41.3 \%$ of the sample. Old age ( $\geq 70$ years) exhibited a significant association with $\mathrm{pT} 4$ $(\mathrm{P}=0.021)$. Body mass index was not evaluated in the majority of the cases; however, from review of the clinical records, obesity was observed in $\sim 20 \%$ of the patients.

A confirmed $\mathrm{FH}$ of $\mathrm{BC}$ was obtained in 30 patients (15.3\%). FH is significantly associated with $\mathrm{G} 2 / \mathrm{G} 3$ carcinomas, high Ki-67, Luminal B-like subtype, high anatomical stage, presence of BRCA2 mutations and bilaterality. A total of 7 patients (3.6\%) exhibited bilateral carcinomas, and 1 patient exhibited synchronous tumors. FH and bilaterality were significantly associated with BRCA2 mutations. Bilaterality was also associated with the presence of NBPN. The occurrence of NBPN was identified in 28 patients (14.3\%). One patient exhibited 3 other carcinomas, in the prostate, colon and kidney, and 2 patients exhibited 2 carcinomas, in the prostate and bladder and in the prostate and kidney. From the remaining patients, 12 had prostate carcinomas, 3 presented with colon-rectal carcinomas, 3 exhibited head and neck squamous cell carcinomas, 3 had gastric carcinomas, 1 had papillary thyroid carcinoma, 1 exhibited chronic lymphocytic leukemia, 1 had Hodgkin disease and 1 exhibited soft tissue histiocytic sarcoma. In the majority of cases, BC was the first neoplasm recorded. In 5 patients, it was the second neoplasm observed; 2 of these patients had exhibited lymphoma previously. A total of 2 patients with prostate carcinoma also had bilateral BC.

A total of 79 patients (40.3\%) presented with pT4 carcinomas and $110(56.4 \%)$ with axillary lymph node metastasis. The majority of the patients $(\mathrm{n}=178 ; 90.8 \%)$ had no distant metastasis at presentation (M0). At diagnosis, 88 patients (44.9\%) were diagnosed with AS II disease. Nodal status and AS were significantly associated with Ki-67. Distant metastases at presentation (M1) were associated with ERBB2-positive carcinomas.

Regarding the histological type, 177 (90.3\%) invasive carcinoma of no special type (NST) were identified, $25 \%$ of which exhibited a range of proportions of in situ components, and the other most frequent invasive subtypes were mucinous carcinoma (1 pure and 4 mixed) and papillary carcinoma (4 cases). The case of pure mucinous carcinoma belonged to a 
Table I. Clinicopathological and therapeutic characteristics of the patient cohort.

\begin{tabular}{|c|}
\hline Characteristics \\
\hline $\begin{array}{l}\text { Age (years) } \\
31-39 \\
40-69 \\
70-89\end{array}$ \\
\hline $\begin{array}{l}\text { Family history }(\mathrm{FH}) \\
\text { No } \\
\text { Yes }\end{array}$ \\
\hline $\begin{array}{l}\text { Bilaterality } \\
\text { No } \\
\text { Yes }\end{array}$ \\
\hline $\begin{array}{l}\text { Non-breast primary } \\
\text { neoplasms (NBPN) } \\
\text { No } \\
\text { Yes }\end{array}$ \\
\hline $\begin{array}{l}\text { Tumor size }(\mathrm{pT}) \\
\text { pTis } \\
\text { pT1 } \\
\text { pT2-3 } \\
\text { pT4 }\end{array}$ \\
\hline $\begin{array}{l}\text { Axillary nodal status } \\
\text { pN0 } \\
\text { pN1 }\end{array}$ \\
\hline $\begin{array}{l}\text { Distant metastasis }(1 \\
\text { M0 } \\
\text { M1 }\end{array}$ \\
\hline $\begin{array}{l}\text { Anatomic stage (AS) } \\
\text { In situ (is) } \\
\text { I } \\
\text { II } \\
\text { III } \\
\text { IV }\end{array}$ \\
\hline
\end{tabular}

Histological type (HT)

In situ
Invasive no special type (NST)
Other invasive subtypes

Histological grade $(\mathrm{G})$

G1

$\mathrm{G} 2$

G3

196

$\mathrm{N} \quad \mathrm{N}(\%)$

196

7 (3.6)

$108(55.1)$

81 (41.3)

$166(84.7)$

$30(15.3)$

196

$189(96.4)$

7 (3.6)

196

$168(85.7)$

28 (14.3)

196

7 (3.6)

52 (26.5)

58 (29.6)

79 (40.3)

195

85 (43.6)

$110(56.4)$

196

$178(90.8)$

18 (9.2)

196

7 (3.6)

77 (39.2)

$88(44.9)$

6 (3.1)

$18(9.2)$

196

6 (3.1)

$177(90.3)$

13 (6.6)

190

45 (23.7)

$110(57.9)$

$35(18.4)$

Therapy

196

177(90.3)

$124(63.3)$

$118(60.2)$

$73(37.2)$

Radiotherapy

Hormonotherapy

Chemotherapy
Table II. Molecular characteristics of the series.

\begin{tabular}{lcc}
\hline Characteristics & $\mathrm{N}$ & $\mathrm{N}(\%)$ \\
\hline BRCA2 mutation & 44 & \\
Indeterminate & & $31(70.5)$ \\
Positive & & $13(29.5)$ \\
Estrogen receptors (ER) & 190 & \\
$\quad$ Positive & & $177(93.1)$ \\
$\quad$ Negative & & $13(6.9)$
\end{tabular}

Progesterone receptors (PR)

190

Positive

$143(75.3)$

Negative

47 (24.7)

ERBB2 (IHC + ISH)

Negative

$179(94.2)$

Positive

$11(5.8)$

Ki67

190

$78(41.1)$

$112(58.9)$

High

Clinical defined subtypes (CS)

190

Luminal A-like

$77(40.5)$

$86(45.3)$

Luminal B-like

$13(6.8)$

HER2-like

$14(7.4)$

DNA ploidy

79

9 (11.4)

$70(88.6)$

$\mathrm{N}$, number of patients; $\mathrm{FH}$, family history; NBPN, non-breast primary neoplasia; pT, tumor size; pN, axillary nodal status; $\mathrm{M}$, distant metastasis; AS, anatomic stage; NST, no special type; G, histological grade.

37 years old patient with no $\mathrm{FH}$, diagnosed with a pT2/pN0/M0 tumor, G2, ER/PR-positive, ERBB2-negative and BRCA indeterminate, who survived with no recurrence during a follow-up of 96 months. A total of 2 patients (1\%), at 51 and 64 years old, were diagnosed with lobular invasive carcinoma, one with a $\mathrm{FH}$ and the other with a pathogenic BRCA2 mutation.

The majority of the male BC cases were graded as G2. A total of 45 cases $(23.7 \%)$ were classified as G1, and only a minority of the cases (18.4\%) was integrated in the G3 group. High grades were associated with high Ki-67 levels.

During the present study, therapeutic strategies for male $\mathrm{BC}$ followed the patterns of the recommendations for $\mathrm{BC}$ in females. The majority of patients (90.3\%) underwent surgery, but only 9 patients (4.6\%) performed breast-conserving surgery. A total of 33 patients (16.8\%) received neoadjuvant therapy. Adjuvant radiotherapy was used in $63.3 \%$ of the patients, adjuvant hormonotherapy in $60.2 \%$ and chemotherapy in $37.2 \%$ of the patients. ERBB2-targeting agents were used in 2 patients in the cohort.

BRCA2 mutations were identified in $13(29.5 \%)$ of the 44 patients examined and, in addition to the associations with FH and bilaterality, were also significantly associated with high Ki-67 and negative PR expression levels. A total of 10 
Table III. Significant associations between clinical and molecular characteristics of the patient cohort (Pearson's chi-square test).

Characteristics
Age $(<40,40-69, \geq 70$ years)
pT
Family history (FH) (no vs. yes)
G
AS
Ki67 index
CS
BRCA2 mutation
Bilaterality (no vs. yes)
FH
Non-breast primary neoplasms (NBPN)
DNA ploidy
BRCA2 mutation

Tumour size (pT) (pT1 vs. pT2-3 vs. pT4)

$\mathrm{pN}$
$\mathrm{M}$
$\mathrm{AS}$
$\mathrm{PR}$

Nodal status (pN) (pN0 vs. pN1)

$\mathrm{M}$

AS

Ki67 index

CS

Distant metastasis (M) (M0 vs. M1)

AS
CS
ERBB2

Anatomic stage (AS) (I vs. II/III vs. IV)

Ki67 index

CS

ERBB2

Histological type (HT) (NST vs. others)

$\mathrm{pT}$
$\mathrm{pN}$
$\mathrm{AS}$

Grade (G) (G1 vs. G2 vs. G3)

$\mathrm{Ki} 67$ index

CS

Estrogen receptors (ER) (positive vs. negative)

PR

Progesterone receptors (PR) (positive vs. negative) BRCA2 mutation

Ki-67 (low vs. high)

CS

ER

PR

ERBB2

BRCA2 mutation

0.021

0.009

0.011

0.002

0.002

0.009

$<0.001$

0.004

0.008

$<0.001$

$<0.001$

$<0.001$

0.029

0.002

$<0.001$

0.003

0.030

$<0.001$

0.030

0.009

0.004

0.009

0.015

$<0.001$

$<0.001$

0.002

$<0.001$

0.010

$<0.001$

0.004

$<0.001$

0.011

0.047
Table III. Continued.

Characteristics

P-value

Age

pT

0.021

Family history (FH)

G

0.009

AS

0.011

$\mathrm{Ki} 67$ index

0.002

CS

0.001

BRCA2 mutation

0.002

0.001 Bilaterality

$\mathrm{FH}$

0.009

Non-breast primary neoplasms (NBPN)

$<0.001$

DNA ploidy

BRCA2 mutation

0.004

0.008

Tumour size (pT)

$\mathrm{pN}$

$<0.001$

$<0.001$

AS

$<0.001$

PR

0.029

Nodal status $(\mathrm{pN})$

$\mathrm{M}$

0.002

AS

$<0.001$

Ki67 index

0.003

CS

0.030

Distant metastasis (M)

AS

$<0.001$

CS

0.030

ERBB2

0.009

Anatomic stage (AS)

Ki67 index

0.004

CS

0.009

ERBB2

0.015

Histological type (HT)

pT

$<0.001$

$\mathrm{pN}$

0.012

AS

$<0.001$

Grade (G)

$<0.001 \quad \mathrm{Ki} 67$ index

$<0.001$

CS

0.002

Estrogen receptors (ER)

PR

$<0.001$

Progesterone receptors (PR)

BRCA2 mutation

0.010

$\mathrm{Ki}-67$

CS

$<0.001$

ER

0.004
PR

$<0.001$

ERBB2

0.011

BRCA2 mutation
FH, Family history; G, Grade; AS, anatomic stage; KI-67, antigen Ki-67; CS, subtype; BRCA2, BRCA2, DNA repair associated; M, Distant metastasis; PR, Progesterone receptors; ERBB2, receptor tyrosine kinase erbB-2; ER, Estrogen receptors. 
(76.9\%) confirmed BRCA2 mutated carcinomas belonged to the surrogate Luminal B-like subtype and 2 cases were HER2-like, according to the AJCC 8th edition classification system (19). No BRCA1 mutations were identified in the series.

The majority of male breast carcinomas were ER-positive/ PR-positive/ERBB2-negative, and 14 (7.4\%) were TN. All PR-positive cases were ER-positive, and 34 cases (75.6\%) of PR-negative carcinomas were ER-positive $(\mathrm{P}<0.0001)$. Using IHC, 35 ERBB2-equivocal (2+) cases and 6 positive (3+) cases were identified. From the equivocal cases, 5 (14.2\%) became positive. In total, 11 ERBB2-positive cases were identified, 10 cases of which were triple-positive and 1 case was ER-positive and PR-negative. Positive ERBB2 expression was significantly associated with M1 carcinomas at presentation, high AS and high Ki-67 expression.

High Ki-67 ( $\mathrm{n}=112 ; 58.9 \%)$ was significantly associated with positive $\mathrm{FH}$, high grades, pN1, high AS, ER-negative, PR-negative and ERBB2-positive expression, and the presence of BRCA2 mutations.

The incidence rates of the 4 clinically-defined CS using IHC, according to AJCC 8th Edition (19), were as follows: Luminal A-like (40.5\%), Luminal B-like (45.3\%), HER2-like (6.8\%) and TN (7.4\%).

DNA ploidy pattern was analyzed in 79 cases, revealing a high percentage of aneuploid carcinomas (88.6\%). As shown in Table III, aneuploid carcinomas were significantly associated with bilaterality.

Survival analysis. The 5 and 10-year DFS rates of patients, excluding patients with M1 carcinomas, patients with non-primary breast neoplasms and in situ carcinomas $(\mathrm{n}=145)$ were 65.9 and $58.2 \%$, respectively, and the 5 and 10 year OS rates were 77.5 and $59.2 \%$, respectively. Mean and median remission times were 75.6 and 50 months (range, 0-312), respectively, and mean and median survival times were 87.8 and 72 months (range, 3-396), respectively. Of the 18 patients with distant metastasis at presentation, only 1 was alive with bone metastasis after 34 months of follow-up. All other 17 patients succumbed to the disease, with the mean and median survival times for all patients with distant metastases (M1) being 18.7 and 15.5 months (range, 1-38 months). The occurrence of NBPN did not decrease OS, as patients with NBPN exhibited 5 and 10-year OS rates of 92.3 and $92.3 \%$ compared with 75.5 and $59.2 \%$ of patients without NBPN.

Kaplan-Meier estimates indicated that a longer DFS and an improved OS were significantly associated with pT1/pN0/stage I (all $\mathrm{P}<0.001)$, low Ki-67 carcinomas $(\mathrm{P}=0.030$ and $\mathrm{P}=0.010$, respectively; Fig. 1 and Tables IV and V), while a shorter DFS and poorer OS were associated with Luminal B-like subtype $(\mathrm{P}=0.002)$ and the presence of BRCA2 mutations ( $\mathrm{P}=0.003$ and $\mathrm{P}<0.001$, respectively) (Fig. 2). Patients with G3 carcinomas also exhibited a shorter DFS ( $\mathrm{P}=0.020)$. Additionally, a longer OS was associated with young age ( $<40$ years; $P=0.010$ and Fig. 3$)$. The patients diagnosed prior to the introduction of taxane chemotherapy exhibited significantly decreased 5 and 10 -year DFS $(\mathrm{P}=0.030)$ and $\mathrm{OS}(\mathrm{P}=0.050)$ compared with those diagnosed in the years following the introduction of taxane chemotherapy.

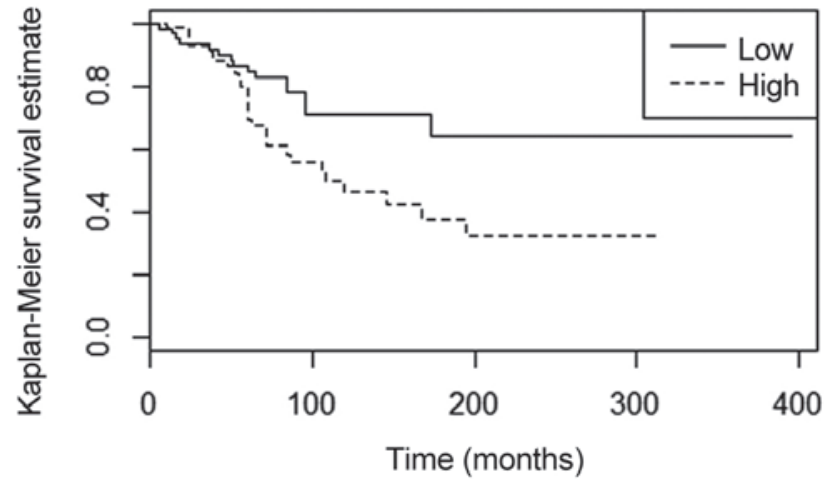

Figure 1. Kaplan-Meier overall survival curve for Ki-67 index. Log rank tests were used to analyze the curves $(\mathrm{P}=0.010)$. Ki-67, antigen Ki-67.

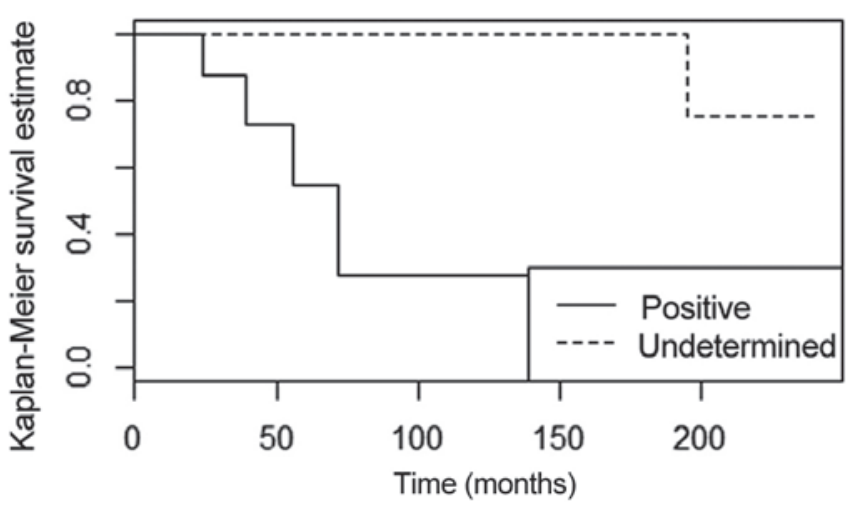

Figure 2. Kaplan-Meier overall survival curve for BRCA2 mutations. Log rank tests were used to analyze the curves $(\mathrm{P}<0.001)$. BRCA2, BRCA2, DNA repair associated.

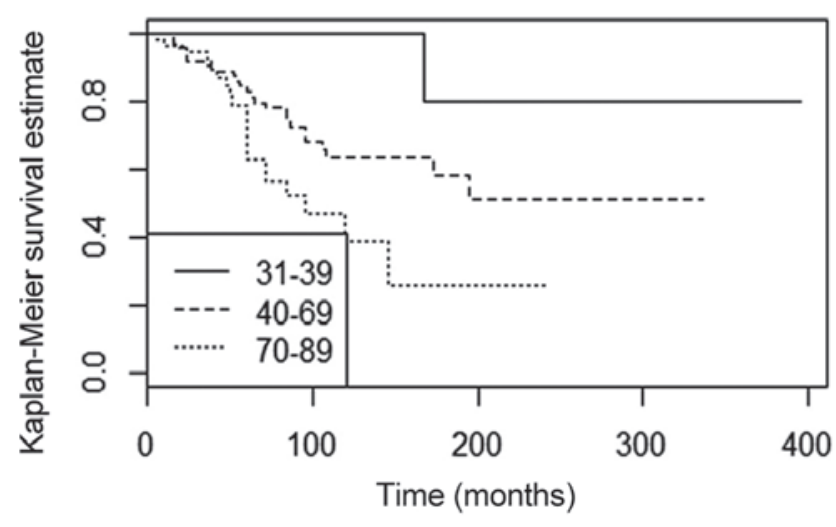

Figure 3. Kaplan-Meier overall survival curve for age (years) of the patients. Log rank tests were used to analyze the curves $(\mathrm{P}=0.010)$.

The results of the univariate Cox model analysis (Table IV) were consistent with the Kaplan-Meier analysis. The categories pT2-3 and 4, pN1, AS II and III, high Ki-67, Luminal B-like and BRCA2 mutations were significantly associated with shorter DFS and OS. In addition, G3 and ages $>70$ years were significantly associated with lower DFS and poorer OS, respectively.

In the multivariate Cox regression analysis (Table V), bilaterality, G3 and AS II and III carcinomas were the 
Table IV. Univariate Cox regression analysis in relation to DFS and OS.

\begin{tabular}{|c|c|c|c|c|c|c|}
\hline \multirow[b]{2}{*}{ Variables } & \multicolumn{3}{|c|}{ Disease-free survival } & \multicolumn{3}{|c|}{ Overall survival } \\
\hline & $\mathrm{RR}$ & $95 \% \mathrm{CI}$ & $\mathrm{P}$ value & $\mathrm{RR}$ & $95 \% \mathrm{CI}$ & P-value \\
\hline \multicolumn{7}{|l|}{ Age (years) } \\
\hline $31-39$ & 1 & - & - & 1 & - & - \\
\hline $40-69$ & 4.37 & $0.59-32.3$ & 0.148 & 4.05 & $0.54-30.1$ & 0.172 \\
\hline $70-89$ & 6.35 & $0.84-48.2$ & 0.074 & 8.02 & $1.06-60.8$ & 0.044 \\
\hline \multicolumn{7}{|l|}{ Grade $(\mathrm{G})$} \\
\hline G1-2 & 1 & - & - & & & \\
\hline G3 & 2.14 & $1.12-4.05$ & 0.020 & & & \\
\hline \multicolumn{7}{|l|}{ Tumor size (pT) } \\
\hline pT1 & 1 & - & - & 1 & - & - \\
\hline pT2-3 & 3.19 & $1.23-8.27$ & 0.017 & 3.55 & $1.14-11.0$ & 0.029 \\
\hline pT4 & 6.10 & $2.50-14.9$ & $<0.001$ & 9.10 & $3.19-26.0$ & $<0.001$ \\
\hline \multicolumn{7}{|l|}{ Nodal status (pN) } \\
\hline pNO & 1 & - & - & 1 & - & - \\
\hline $\mathrm{pN} 1$ & 6.32 & $2.93-13.6$ & $<0.001$ & 6.40 & $2.84-14.4$ & $<0.001$ \\
\hline \multicolumn{7}{|c|}{ Anatomic stage (AS) } \\
\hline I & 1 & - & - & 1 & - & - \\
\hline II & 6.71 & $3.11-14.5$ & $<0.001$ & 7.81 & $3.28-18.6$ & $<0.001$ \\
\hline III & 4.80 & $1.01-22.7$ & 0.048 & 11.1 & $2.75-44.9$ & $<0.001$ \\
\hline \multicolumn{7}{|l|}{ Ki-67 } \\
\hline Low & 1 & - & - & 1 & - & - \\
\hline High & 1.89 & $1.05-3.39$ & 0.033 & 2.14 & $1.16-3.96$ & 0.015 \\
\hline \multicolumn{7}{|c|}{ Clinical subtype (CS) } \\
\hline Luminal A-like & 1 & - & - & 1 & - & - \\
\hline Luminal B-like & 2.67 & $1.45-4.90$ & 0.002 & 2.97 & $1.58-5.60$ & $<0.001$ \\
\hline HER2-like & 0.81 & $0.11-6.08$ & 0.837 & 1.25 & $0.16-9.51$ & 0.828 \\
\hline Triple negative & 0.52 & $0.12-2.23$ & 0.379 & 0.64 & $0.15-2.80$ & 0.556 \\
\hline \multicolumn{7}{|l|}{ BRCA2 mutation } \\
\hline Indeterminate & 1 & - & - & 1 & - & - \\
\hline Positive & 0.11 & $0.02-0.63$ & 0.013 & 0.06 & $0.01-0.52$ & 0.011 \\
\hline
\end{tabular}

DFS, disease-free survival; pT, tumor size; pN, nodal status; M, distant metastasis; AS, anatomic stage; G, grade; Ki-67, antigen Ki-67; CS, clinical subtype; BRCA2, BRCA2, DNA repair associated; HER2, receptor tyrosine kinase erbB-2; TN, triple negative.

significant factors associated with a higher risk of disease recurrence. The presence of FH, AS II and III and Luminal B-like subtype were the significant characteristics associated with low OS.

\section{Discussion}

$\mathrm{BC}$ is a complex disease that affects females and males, and the primary known difference between sexes is incidence. According to recognized biological heterogeneity, studies comparing female and male $\mathrm{BC}$ have demonstrated similarities and differences $(1,3,7,30,31)$. The understanding of the effects of the clinicopathological, molecular and genomic features associated with therapy and prognosis is progressing continuously in male $\mathrm{BC}$ and, as more data become available, the hypothesis that males only exhibit endocrine-associated
$\mathrm{BC}$ identical to that in postmenopausal females becomes less plausible, and male BC emerges as a distinctive subtype of BC lacking its own guidelines (15,32-36).

Survival has been a controversial issue in male BC. The majority of studies have demonstrated a poorer outcome in male compared with female patients, but others revealed that there was no difference in the prognosis of the two sexes, when paired according to specific groups (15,30,32-38). A similar DFS and OS to pre/peri-menopausal females, but poorer compared with post-menopausal female $\mathrm{BC}$, was described in male patients with $\mathrm{BC}$ (32), and even a lower risk of mortality compared with comparable females, despite the frequent presentation in elderly and more advanced disease in male $\mathrm{BC}$ (1). M1 patients have incurable disease and, in the patient cohort in the present study, all but 1 succumbed to the disease, with a median survival time of 15.5 months. The present study 
Table V. Multivariate Cox regression analysis in relation to DFS and OS.

\begin{tabular}{|c|c|c|c|c|c|c|}
\hline \multirow[b]{2}{*}{ Variables } & \multicolumn{3}{|c|}{ Disease-free survival } & \multicolumn{3}{|c|}{ Overall survival } \\
\hline & $\mathrm{RR}$ & $95 \% \mathrm{CI}$ & P-value & $\mathrm{RR}$ & $95 \% \mathrm{CI}$ & P-value \\
\hline \multicolumn{7}{|l|}{ Bilaterality } \\
\hline No & 1 & - & - & & & \\
\hline Yes & 6.16 & $1.30-29.3$ & 0.022 & & & \\
\hline \multicolumn{7}{|c|}{ Family history (FH) } \\
\hline \multicolumn{7}{|l|}{ No } \\
\hline Yes & & & & 0.33 & $0.13-0.90$ & 0.030 \\
\hline \multicolumn{7}{|l|}{ Grade $(\mathrm{G})$} \\
\hline G1-2 & 1 & - & - & 1 & - & - \\
\hline G3 & 2.20 & $1.10-4.42$ & 0.026 & 2.06 & $1.0-4.24$ & 0.051 \\
\hline \multicolumn{7}{|l|}{ Anatomic stage } \\
\hline I & 1 & - & - & 1 & - & - \\
\hline II & 4.08 & $1.53-10.9$ & 0.005 & 8.95 & $3.65-21.9$ & $<0.001$ \\
\hline III & 6.79 & $1.15-40.2$ & 0.035 & 45.7 & $9.92-211$ & $<0.001$ \\
\hline \multicolumn{7}{|l|}{ Clinical subtype } \\
\hline Luminal A-like & 1 & - & - & 1 & - & - \\
\hline Luminal B-like & 1.72 & $0.92-3.23$ & 0.091 & 2.05 & $1.07-3.93$ & 0.030 \\
\hline
\end{tabular}

OS, overall survival; pT, tumor size; pN, nodal status; M, distant metastasis; AS, anatomic stage; G, grade; Ki-67, antigen Ki-67; CS, clinical subtype; PR, progesterone receptor; BRCA2, BRCA2, DNA repair associated; HER2, receptor tyrosine kinase erbB-2; TN, triple negative.

encompassed a long time period, with the 10 -year OS rates of cases (stages I-III; 59.2\%) measuring slightly longer compared with those demonstrated by Leone et al (53.7\%) (6), Chen et al (40.1\%) (38) and Tural et al (52.5\%) (39).

The risk of developing $\mathrm{BC}$ increases with age, similar to the majority of carcinomas at all sites. In the present study, the percentage of the patients aged $\geq 70$ years $(41.3 \%)$ confirms the high incidence of $\mathrm{BC}$ in older males, and also that the average age at diagnosis is $~ 5-10$ years older compared with in females $(1,12,36)$. The high frequency in the elderly population is important, as the therapeutic approach in older male patients is based on studies performed in females of different ages, and comorbidities in the elderly population may result in inadequate treatment. In the present study, elderly patients exhibited larger carcinomas and higher $\mathrm{Ki}-67$ expression levels compared with younger patients, and old age was a prognostic factor significantly associated with low 5 and 10-year OS in Kaplan-Meier estimates, which were concordant with data from previous studies $(6,7,33,38,39)$. Poorer prognoses in older males may be associated with tumor biology, late diagnosis, comorbidities and/or inadequate therapeutic management, and constitutes a persistent clinical problem $(33,39)$. Similar to older patients, obese patients have unknown risk factors affecting the accurate prediction of toxicity of treatments and prognosis (35). Obesity is an important risk factor and the proportion of obese male patients with $\mathrm{BC}$ observed in the present study was similar to that identified by Gargiulo et al $(8,40)$.

FH appears to be particularly relevant in male $\mathrm{BC}$. Bouchardy et al (41) identified a positive $\mathrm{FH}$ in $20 \%$ of male patients with $\mathrm{BC}$, but no significant differences in OS in patients with FH compared with sporadic cases were observed. As the present study included patients diagnosed from 1970 onwards, the majority of patients had no information regarding $\mathrm{FH}$ in their clinical records. However, a confirmed family history of $\mathrm{BC}$ in a first-degree relative was significantly associated with BRCA mutations, and also to high AS, high grade, high Ki-67 and Luminal B-like subtype. Additionally, in the multivariate analysis, a positive $\mathrm{FH}$ was associated with OS. In concordance with previous data (41), positive $\mathrm{FH}$ was also associated with bilateral male BC. Bilaterality occurred in $3.6 \%$ of the patients in the cohort within the present study, and was significantly associated with BRCA2 mutations and with the presence of NBPN. Male patients with BC also have an increased risk of NPBN, and the long survival times currently observed should be observed cautiously (42-44). A total of $\sim 14 \%$ of the patients in the present study exhibited NBPN, and 2 with bilaterality and prostate carcinoma. As described previously (45), prostate carcinoma is the most frequently observed non-breast primary tumor. The risk of head and neck, colon and thyroid carcinomas were demonstrated to be high in male BC $(43,44)$, and their occurrence was also observed in the present study. A total of 2 patients had previous lymphoma, supporting the observation that males who survive lymphoma may have an increased risk of developing BC $(44,46)$. Among the factors identified to be responsible for causing a second neoplasia, genetic factors appear to represent an important contribution. These data suggest the requirement of a genetic consultation in male $\mathrm{BC}$. BRCA2 is one of the most frequently mutated genes in male $\mathrm{BC}$, ranging between 4 and $40 \%$ depending on the population studied (15); $29.5 \%$ of the 44 patients included in the present study exhibited this mutation, while BRCA1 mutations are infrequent; none were observed in the present study, suggesting 
a dissimilar genetic etiology between sexes (13). As described previously $(8,16)$, the majority of BRCA2-mutated carcinomas in the present study belonged to the Luminal B-like subtype, and a significant association between BRCA2 mutations and poorer prognosis was observed.

The AS classification systems represents one of the most important established prognostic tools for male BC, as demonstrated in the present study and in previous studies $(6,8,34)$. Male BC is increasingly diagnosed earlier $(6,7)$, and a predominance of early stages was observed in the present study. However, high rates of advanced stages are frequently observed $(1,6,8,12,33,36,37)$. The unviability of screening due to low incidence rates, the high occurrence of gynecomastia that may exhibit identical presentation symptoms, the fact that males are less likely to report symptoms that would lead to early diagnosis, the absence of publicly-available information about the disease, the incidence in old age with an associated suboptimal access to healthcare, and anatomic and biological differences, may explain the number of diagnoses at high stages observed $(4,36,39)$.

The proportion of pure in situ carcinomas, one-half with papillary morphology, varies in previous studies, but is significantly decreased compared with the proportion described in females $(30,33,47)$. The relative frequency of papillary morphology, either in situ or invasive carcinomas, may be associated with the common subareolar localization in male BC. The heterogeneous histological type of invasive carcinoma NST, with an occurrence between 85 and $95 \%$ described in previous studies $(5,37)$, was diagnosed in $90.3 \%$ of cases in the present study. The percentage of associated in situ components was similar to the proportion demonstrated in females (48). Mucinous carcinoma accounts for $1-4 \%$ of male $\mathrm{BC}$, has a favorable prognosis in the pure form, but the pathogenesis is not understood $(30,39,49,50)$. In the present study, 5 cases (2.5\% of all cases) were observed, one being the patient with a pure form, unusually young for the described in mucinous carcinomas. Invasive lobular carcinoma, the second most frequent histological type in females (10\% of the cases), is exceptionally rare in males (1\%) and its etiology remains unexplained considering the lack of development of terminal lobules in males $(6,51)$. A total of 2 invasive lobular carcinomas (1\%) were identified in the present study, both with negative epithelial-cadherin staining.

G2 carcinomas were predominant in the present study, similar to other previous studies $(5,7,30,33,52)$. High histological grade (G3) is commonly associated with poor prognosis, but this is not always statistically significant $(6,7)$. In the present study, G3 carcinomas occurred in $18.4 \%$ of the cases, and were demonstrated to be significantly associated with FH, high Ki-67 expression, Luminal B clinical subtype and poorer prognosis in univariate and multivariate Cox regression analyses.

The lack of randomized trials in male BC explains why therapy is based on the guidelines for $\mathrm{BC}$ in females. However, due to primarily anatomical and hormonal reasons, the management is not exactly the same, highlighting the requirement to improve the personalized care of male BC $(17,38)$. Breast-conserving surgery vs. mastectomy may be performed in early stages, but is rarely used due to the paucity of breast tissue and the frequent subareolar location of carcinomas associated with the distribution of epithelial breast tissue $(6-8,49)$. Tamoxifen is the most frequently employed systemic treatment $(38,49)$, but low tolerance, side effects and high rates of discontinuation have been described $(4,17,38,49)$. The relatively low rate of hormonotherapy compared with the high percentage of ER-positive carcinomas identified in the present study, and demonstrated in previous studies $(7,31)$, may be associated with the fact that the use of tamoxifen in males was only recently standardized (7). Different chemotherapy agents and regimens have been used and the introduction of taxanes marked a significant advance in the treatment of metastatic disease in females, but there are no specific evidence-based guidelines for male BC $(33,35,49)$. In addition to the therapeutic effects of the treatment, the improvement in DFS and OS observed in the present study when comparing the groups of patients diagnosed prior and subsequent to taxane chemotherapy may be associated with early diagnosis, standardized clinicopathological evaluation and improved follow-up observed in recent years (1).

Biomarker evaluation by IHC has resulted in differing data among male BC studies, primarily due to different methodologies, the development of scoring systems and the range of cut-off values used, but the high frequency of ER-positive/PR-positive expression and the low frequency of $\mathrm{TN}$ carcinomas are concordant $(7,8,38,45,52)$. ER-positive expression is associated with improved prognoses at 5 -year OS (6), but certain clinically aggressive male BC cases do not appear to have an active ER pathway (16). In the present study, negative PR expression status was associated with BRCA mutations.

Despite the different estimates described, ERBB2 positivity has a low frequency in males $(7,51)$. Using IHC and ISH, 6.8\% of the cases in the present study were identified as HER2-like clinical subtypes, according to AJCC 8th Edition (19), and significantly associated with a high Ki-67 expression level and high AS. ERBB2 positivity is generally associated with aggressive phenotypes, but survival of HER2-like carcinomas has improved in previous years, due to specific treatment with associated ERBB2-targeting agents $(19,33)$.

Cell proliferation is also an important biological factor, usually associated with poor outcome (53). Ki-67, a nuclear protein present during all phases of the cell cycle, is the most commonly used marker to evaluate proliferation, although it lacks standardized methodology or generally accepted cut-offs. With a cut-off of $\geq 20 \%$, previous studies identified a predominance of low $\mathrm{Ki}-67$ values $(7,8)$. By contrast, the present study identified a slight predominance of high Ki-67 values, significantly associated with old age, positive FH, high grade, pN1, high AS, CS and poor prognosis in the univariate Cox regression analysis.

The criteria for the definition of $\mathrm{BC}$ molecular subtypes used clinically for decisions regarding therapy are continuously progressing, resulting in difficulties when comparing data $(5,7,15,30,38,52)$. Using IHC surrogates, Luminal A-like and Luminal B-like subtypes were identified in the majority of $\mathrm{BC}$ in males and females, usually with a poorer outcome for Luminal B-like $(7,15,26)$. As PR, ERBB2 and Ki-67 are important prognostic and predictive factors, the inclusion of carcinomas with positive or negative PR and/or ERBB2 expression statuses and different $\mathrm{Ki}-67$ cut-offs in the same subtype are key factors contributing to discordant results. 
In the present study, according to the AJCC 8th edition (19), Luminal B-like subtype exhibited the poorest OS.

Bezić et al (54) observed aneuploidy in $78 \%$ of 31 male patients with BC. In a previous comparative study between sexes (50 cases each), our group demonstrated a significantly higher frequency of DNA aneuploid tumors in males compared with females (80 vs. 46\%) (55). The high percentage of aneuploidy, which was observed to be increased in the present study, suggested a distinctive genomic instability in the carcinogenesis of male $\mathrm{BC}$.

The present study has the limitations of a retrospective study from a single institution conducted over a long time period. However, the results are consistent with those of large and/or multi-institutional studies, confirming that studies involving smaller, but well-characterized clinicopathological and molecular subgroups, diagnosed and followed in multidisciplinary departments within single institutions, are important in improving the understanding of this disease.

In conclusion, the present study demonstrated that male BC was more likely to be diagnosed in older patients (with consequent associated comorbidities and suboptimal therapy), and exhibited poorer prognosis in elderly and in high anatomical stages. BRCA2 mutations were frequent, associated with $\mathrm{FH}$, bilaterality, high $\mathrm{Ki}-67$, PR negativity and Luminal B-like subtype, and with shorter DFS and OS in univariate analysis. In addition, male patients with $\mathrm{BC}$ were at high risk for NBPN. In the multivariate analysis, FH and Luminal B carcinomas were associated with poorer OS. These data underline the importance of early diagnosis and genetic screening in male BC. As sex may be a crucial feature to improve personalized care, additional studies investigating male BC are warranted and may lead to the development of relevant management approaches for BC in males and females.

\section{Acknowledgements}

Not applicable.

\section{Funding}

Professor Giovani Silva was partially funded by FCT-Portugal project (grant no. UID/MAT/00006/2019).

\section{Availability of data and materials}

All data generated or analyzed during this study are included in this published article.

\section{Authors' contributions}

SA and AEP discussed experimental design, interpreted and discussed the data and wrote the manuscript. TP and FS performed IHC experiments. PM and FV performed BRCA analysis. MA and GLS analyzed and interpreted statistical data.

\section{Ethics approval and consent to participate}

The present study was approved by the Institutional Ethics Committee of the Portuguese Institute of Oncology Lisbon Center.

\section{Patient consent for publication}

Not applicable.

\section{Competing interests}

The authors declare that they have no competing interests.

\section{References}

1. Miao H, Verkooijen HM, Chia KS, Bouchardy C, Pukkala E, Larønningen S, Mellemkjaer L, Czene $\mathrm{K}$ and Hartman M: Incidence and outcome of male breast cancer: An international population-based study. J Clin Oncol 29: 4381-4386, 2011.

2. Speirs V and Shaaban AM: The rising incidence of male breast cancer. Breast Cancer Res Treat 115: 429-430, 2009.

3. Ly D, Forman D, Ferlay J, Brinton LA and Cook MB: An international comparison of male and female breast cancer incidence rates. Int J Cancer 132: 1918-1926, 2013.

4. White J, Kearins O, Dodwell D, Horgan K, Hanby AM and Speirs V: Male breast carcinoma: Increased awareness needed. Breast Cancer Res 13: 219, 2011.

5. Yalaza M, İnan A and Bozer M: Male breast cancer. J Breast Health 12: 1-8, 2016.

6. Leone JP, Zwenger AO, Iturbe J, Leone J, Leone BA, Vallejo CT and Bhargava R: Prognostic factors in male breast cancer: A population-based study. Breast Cancer Res Treat 156: 539-548, 2016.

7. Cardoso F, Bartlett JMS, Slaets L, van Deurzen CHM, van Leeuwen-Stok E, Porter P, Linderholm B, Hedenfalk I, Schröder C, Martens J, et al: Characterization of male breast cancer: Results of the EORTC 10085/TBCRC/BIG/NABCG international male breast cancer program. Ann Oncol 29: 405-417, 2018.

8. Gargiulo P, Pensabene M, Milano M, Arpino G, Giuliano M, Forestieri V, Condello C, Lauria R and De Placido S: Long-term survival and BRCA status in male breast cancer: A retrospective single-center analysis. BMC Cancer 16: 375, 2016.

9. Anderson WF, Jatoi I, Tse J and Rosenberg PS: Male breast cancer: A population-based comparison with female breast cancer. J Clin Oncol 28: 232-239, 2010.

10. Miranda AC: National Oncological Registry South (ROR-South) 2010-2011. Incidence, Survival and Mortality for cancer in the Southern region of Portugal - ISM2010/2011. ROR-South. Portuguese Institute of Oncology of Lisbon, 2017.

11. Kreiter E, Richardson A, Potter J and Yasui Y: Breast cancer: Trends in international incidence in men and women. $\mathrm{Br}$ J Cancer 110: 1891-1897, 2014.

12. Ferzoco RM and Ruddy KJ: The epidemiology of male breast cancer. Curr Oncol Rep 18: 1, 2016.

13. Deb S, Lakhani SR, Ottini L and Fox SB: The cancer genetics and pathology of male breast cancer. Histopathology 68: 110-118, 2016.

14. Pritzlaff M, Summerour P, McFarland R, Li S, Reineke P, Dolinsky JS, Goldgar DE, Shimelis H, Couch FJ, Chao EC and LaDuca H: Male breast cancer in a multi-gene panel testing cohort: Insights and unexpected results. Breast Cancer Res Treat 161: 575-586, 2017.

15. Johansson I, Killander F, Linderholm B and Hedenfalk I: Molecular profiling of male breast cancer-lost in translation? Int J Biochem Cell Biol 53: 526-535, 2014.

16. Keinan-Boker L, Levine H, Leiba A, Derazne E and Kark JD: Adolescent obesity and adult male breast cancer in a cohort of 1,382,093 men. Int J Cancer 142: 910-918, 2018.

17. Khan MH, Allerton R and Pettit L: Hormone therapy for breast cancer in men. Clin Breast Cancer 15: 245-50, 2015.

18. Pemmaraju N, Munsell MF, Hortobagyi GN and Giordano SH: Retrospective review of male breast cancer patients: Analysis of tamoxifen-related side-effects. Ann Oncol 23: 1471-1474, 2012.

19. Amin MB, Edge SB, Greene FL, Byrd DR, Brookland RK, Washington MK, Gershenwald JE, Compton CC, Hess KR, Sullivan DC, et al (eds): AJCC cancer staging manual, eighth edition, 2017.

20. Lakhani SR, Ellis IO, Schnitt SJ, Hoon Tan PH and van de Vijver MJ, eds: World health organization classification of tumors of the breast. lyon: IARC; WHO Classification of Tumours, 2012. 
21. Elston CW and Ellis IO: Pathological prognostic factor in breast cancer. I. the value of histological grade in breast cancer: Experience from a large study with long follow-up. Histopathology 19: 403-410, 1991.

22. Machado PM, Brandão RD, Cavaco BM, Eugénio J, Bento S, Nave M, Rodrigues P, Fernandes A and Vaz F: Screening for a BRCA2 rearrangement in high-risk breast/ovarian cancer families: Evidence for a founder effect and analysis of the associated phenotypes. J Clin Oncol 25: 2027-2034, 2007.

23. Freitas AC, Opinião A, Fragoso S, Nunes H, Santos M, Clara A, Bento S, Luís A, Silva J, Moura C, et al: Men seeking counselling in a breast cancer risk evaluation clinic. Ecancermedicalscience 12: 804, 2018.

24. Hammond ME, Hayes DF, Wolff AC, Mangu PB and Temin S: American Society of Clinical Oncology/College of American Pathologists guideline recommendations for immunohistochemical testing of estrogen and progesterone receptors in breast cancer. J Oncol Pract 6: 195-197, 2010.

25. Wolff AC, Hammond ME, Hicks DG, Dowsett M, McShane LM, Allison KH, Allred DC, Bartlett JM, Bilous M, Fitzgibbons $\mathrm{P}$, et al: Recommendations for human epidermal growth factor receptor 2 testing in breast cancer: American society of clinical oncology/college of American pathologists clinical practice guideline update. J Clin Oncol 31: 3997-4013, 2013.

26. Goldhirsch A, Winer EP, Coates AS, Gelber RD, Piccart-Gebhart M, Thürlimann B and Senn HJ; Panel members: Personalizing the treatment of women with early breast cancer: Highlights of the St gallen international expert consensus on the primary therapy of early breast cancer 2013. Ann Oncol 24: 2206-2223, 2013.

27. Hedley DW, Friedlander ML, Taylor IW, Rugg CA and Musgrove EA: Method for analysis of cellular DNA content of paraffin-embedded pathological material using flow cytometry. J Histochem Cytochem 31: 1333-1335, 1983.

28. Schäler J, Thaller G, Hinrichs D and R Core Team R: A Language and environment for statistical computing. R Foundation for statistical computing. Vienna, Austria. Agricultural Sciences, Vol. 9, 2018.

29. Akaike H: A new look at the statistical model identification. IEEE Transactions on Automatic Control 19: 716-723, 1974.

30. Shaaban AM, Ball GR, Brannan RA, Cserni G, Di Benedetto A, Dent J, Fulford L, Honarpisheh H, Jordan L, Jones JL, et al: Characterization of male breast cancer: Results of the EORTC 10085/TBCRC/BIG/NABCG international male breast cancer program. Ann Oncol 29: 405-417, 2018.

31. Greif JM, Pezzi CM, Klimberg VS, Bailey L and Zuraek M: Gender differences in breast cancer: Analysis of 13,000 breast cancers in men from the national cancer data base. Ann Surg Oncol 19: 3199-3204, 2012.

32. Yu XF, Yang HJ, Yu Y, Zou DH and Miao LL: A prognostic analysis of male breast cancer (MBC) compared with post-menopausal female breast cancer (FBC). PLoS One 10: e0136670, 2015.

33. Wu Q, Li J, Zhu S, Wu J, Li X, Liu Q, Wei W and Sun S: Poorer breast cancer survival outcomes in males than females might be attributable to tumor subtype. Oncotarget 7: 87532-87542, 2016.

34. Rushton M, Kwong A, Visram H, Graham N, Petrcich W and Dent S: Treatment outcomes for male breast cancer: A single-centre retrospective case-control study. Curr Oncol 21: e400-e407, 2014.

35. Yu E, Stitt L, Vujovic O, Joseph K, Assouline A, Younus J, Perera F and Tai P: Male breast cancer prognostic factors versus female counterparts with propensity scores and matched-pair analysis. Cureus 7: e355, 2015.

36. Gnerlich JL, Deshpande AD, Jeffe DB, Seelam S, Kimbuende E and Margenthaler JA: Poorer survival outcomes for male breast cancer compared with female breast cancer may be attributable to in-stage migration. Ann Surg Oncol 18: 1837-1844, 2011.
37. Li X, Yang J, Krishnamurti U, Huo L, Ward KC, O'Reagan R and Peng L: Hormone receptor positive breast cancer has a worse prognosis in male than in female patients. Clin Breast Cancer 17: 356-366, 2017

38. Chen X, Liu X, Zhang L, Li S, Shi Y and Tong Z: Poorer survival of male breast cancer compared with female breast cancer patients may be due to biological differences. Jpn J Clin Oncol 43: 954-963, 2013.

39. Tural D, Ukbiricik F, Aydogan F, Bese N, Yetmen O, Ilvan S, Buyukunal E and Sergendeçti S: Male breast cancers behave differently in elderly patients. Jpn J Clin Oncol 43: 22-27, 2013.

40. Freedman RA and Partridge AH: Emerging data and current challenges for young, old, obese, or male patients with breast cancer. Clin Cancer Res 23: 2647-2654, 2017.

41. Bouchardy C, Rapiti E, Fioretta G, Schubert H, Chappuis P, Vlastos $G$ and Benhamou S: Impact of family history of breast cancer on tumor characteristics, treatment, risk of second cancer and survival among men with breast cancer. Swiss Med Wkly 143: w13879, 2013.

42. Zheng G, Yu H, Hemminki A, Försti A, Sundquist K and Hemminki K: Familial associations of male breast cancer with other cancers. Breast Cancer Res Treat 166: 897-902, 2017.

43. Hemminki K, Scélo G, Boffetta P, Mellemkjaer L, Tracey E, Andersen A, Brewster DH, Pukkala E, McBride M, Kliever EV, et al: Second primary malignancies in patients with male breast cancer. Br J Cancer 92: 1288-1292, 2005.

44. Hung MH, Liu CJ, Teng CJ, Hu YW, Yeh CM, Chen SC, Chien SH, Hung YP, Shen CC, Chen TJ, et al: Risk of second non-breast primary cancer in male and female breast cancer patients: A population-based cohort study. PLoS One 11: e0148597, 2016.

45. Masci G, Caruso M, Caruso F, Salvini P, Carnaghi C, Giordano L, Miserocchi V, Losurdo A, Zuradelli M, Torrisi R, et al: Clinicopathological and immunohistochemical characteristics in male breast cancer: A retrospective case series. Oncologist 20: 586-592, 2015.

46. Farr DE, Thomas A, Khan SA and Schroeder MC: Male breast cancer as a second primary cancer: Increased risk following lymphoma. Oncologist 22: 895-900, 2017.

47. Brents $M$ and Hancock J: Ductal carcinoma in situ of the male breast. Breast Care (Basel) 11: 288-290, 2016.

48. Kuhl CK, Strobel K, Bieling H, Wardelmann E, Kuhn W, Maass N and Schrading S: Impact of preoperative breast MR imaging and MR-guided surgery on diagnosis and surgical outcome of women with invasive breast cancer with and without DCIS component. Radiology 284: 645-655, 2017.

49. Bradley KL, Tyldesley S, Speers CH, Woods R and Villa D: Contemporary systemic therapy for male breast cancer. Clin Breast Cancer 14: 31-39, 2014.

50. Ishida M, Umeda T, Kawai Y, Mori T, Kubota Y, Abe H, Iwai M, Yoshida K, Kagotani A, Tani T and Okabe H: Mucinous carcinoma occurring in the male breast. Oncol Lett 7: 378-380, 2014.

51. Senger JL, Adams SJ and Kanthan R: Invasive lobular carcinoma of the male breast-a systematic review with an illustrative case study. Breast Cancer (Dove Med Press) 9: 337-345, 2017.

52. Abreu MH, Afonso N, Abreu PH, Menezes F, Lopes P, Henrique R, Pereira D and Lopes C: Male breast cancer: Looking for better prognostic subgroups. Breast 26: 18-24, 2016.

53. Nilsson C, Koliadi A, Johansson I, Ahlin C, Thorstensen S, Bergkvist L, Hedenfalk I and Fjallskog ML: High proliferation is associated with inferior outcome in male breast cancer patients. Mod Pathol 26: 87-94, 2013.

54. Bezić J, Šamija Projić I, Projić P, Ljubkovic J, Zekic Tomas S, Meljanac Salopek K, Pilgic Burazer M and Tomic S: Flow cytometric DNA hipertetraploid tends to be more frequent in male than in female breast cancers. Virchows Arch 466: 185-189, 2015.

55. André S, Pinto AE, Laranjeira C, Quaresma $M$ and Soares J: Male and female breast cancer-differences in DNA ploidy, p21 and 553 expression reinforce the possibility of distinct pathways of oncogenesis. Pathobiology 74: 323-327, 2007. 